\title{
ACTIVE TREMOR COMPENSATION FOR LASER MEDICAL INSTRUMENTS
}

\author{
Emil-Ionut Niță ${ }^{1}$, Philip Coandăa $\breve{1}^{1}$ Constantin-Daniel Comeagă $\breve{a}^{1}$, Mircea Udrea $^{2}$ \\ ${ }^{1}$ University "Politehnica" of Bucharest \\ ${ }^{2}$ Apel Laser S.R.L \\ Emails:nitaemilionut@yahoo.com; philip.coanda@upb.ro; comeaga_daniel@yahoo.com; \\ mircea.udrea@apellaser.ro
}

\begin{abstract}
This paper wishes to provide an overview for active tremor compensations methods for laser medical instruments, considering the pure mechanical and the optical approaches. Moreover, the tremor problematics is approached, considering that in all its forms, tremor can have an impact in one's life. Of course, the main impact on tremor is underlined in microsurgery, where enhanced positioning and precision are needed in order to provide high quality medical act. An overview of the literature is presented, where many solutions can be seen. The principal solutions treated in this paper are the pure mechanical one, which aims to achieve tremor compensation by using different translations and rotations for the laser source, all integrated in a custom design and the optical one, which takes advantage of well know optic principles in order to compensate the tremor. Both methods are analysed through this paper, both advantages and disadvantages for each method being underlined.
\end{abstract}

Keywords: Tremor, Compensations, Laser, Medical, Optical, Active.

\section{Tremor Problematics}

Not observed during daily life activities, tremor, in all its forms, can have an impact on one's life. The most relevant examples where tremor is mentioned are the imaging sessions (CT, MRI) where it can alter the procedure result. Tremor is an unvoluntary, oscillatory and rhythmic movement which is produced by reciprocally innervated antagonist muscles [1][2]. Two broad classifications systems are used in literature, one based of the occurring moment of the tremor, resting or action, and the other one based on cause, were we can encounter multiple causes, phycological and pathological. The tremors occurring during movement are also divided into postural tremor, which occurs during maintained posture, kinetic tremor, which occurs during movement from point to point, task-specific tremor, which occurs during execution of specific tasks and isometric tremor, which occurs during a voluntary muscle contraction and an opposition of a rigid, stationary object [3].

Small amplitude tremor which comes together with normal posture and movement is called physiologic tremor [4]. If we consider the movement disorders which are involuntary, rhythmic oscillatory and imply one more region of the body, they are labelled as pathologic tremor. The differences between multiple types of tremors are based on criteria as frequency and amplitude - low, medium, high. Yet not being clear what is the real purpose of these movements, as per study [5], the function of the physiological tremor is considered to be a solution to keep the motor system oscillating, this way being able to time the motor events.

The tremor represents also one of the main problems considered in manual operations. These unwanted movements of the body can cause major problems when performing critical operations. For example, paper [6] underline the fact that the psychological hand tremor which covers the $8-12 \mathrm{~Hz}$ frequency band and $50 \mu \mathrm{m}$ amplitude peak to peak, can cause serious problems for microsurgeons who are requested precisions of $100 \mu \mathrm{m}$ at least. Brain tumours removal and ophthalmological microsurgery [7] requests high precisions considering the critical zones of operations and even a minor presence of tremor could have a major impact. Multiple techniques for tremor attenuation are considered, as wrist rests and supports. Caffeine limitation and regulated sleep schedule prior to surgery may also be options considered for microsurgeons to limit the tremor [8]. Multiple papers and studies treat the tremor problem in microsurgery, having a big impact on quality of medical act for both patient and doctor.

Considering all the aforementioned aspects, the study of tremor and practical ways of attenuating is a continuous and ongoing work. Tremor has a broad action area, from everyday life to specific areas and tasks. Further analysis will be performed during this paper. 


\section{Existing Concepts and Solutions}

There are many devices specially created for hand tremor compensation based on different levels of tremor amplitude, varying form hand shaking disorders to physiological tremor. In the case of severe tremor disorders like Parkinson that causes problems in daily activities like holding a glass of water or using cutlery, there are a few patents that can help these patients to feel more confident and comfortable. Devices like wearable exoskeletons [9] can be included in the mechatronics field and may help people with limb weaknesses or injury to live a normal life. Some of the well-known exoskeleton applications is WOTAS [10] (wearable orthosis for tremor assessment and suppression), a structure that combines biomechanics and biomechatronic into a wearable medical device that is able to apply forces in specific points to cancel tremor, helping the patients to move and control the limb/arm as they desire. Another device found in literature using DC gear motors with a feedback loop position was used to reduce pathological hand tremor.

Assuming the tremor frequency is in a $3-15 \mathrm{~Hz}$ range and the daily activities should not exceed $3 \mathrm{~Hz}$ in frequency, a suppression hand tremor technique may be applied using the feedback position from the DC gear motors. Different suppression methods of pathological hand tremor may consist in special hand tools designed with an active cancelling actuating system [11]. A more in-depth research reveals examples of finger tremor suppression devices such as WTSG [12] (Wearable Tremor Suppression Glove) that uses inertial measurement units to detect the hand movement and a microcontroller to estimate the actual tremor. These devices can be used to mechanically suppress the parkinsonian tremor of the hand without affecting the voluntary motion.

The results obtained in these cases, where the patients suffer from pathological conditions, represent the starting point to a new more complex research with applicability in the medical field. Moving forward and considering the natural tremor of a person as an input for a tremor cancelling system, the theoretical design approach changes due to the fact that the tremor amplitude reduces down to the micrometric level (almost $100 \mu \mathrm{m}$ peak to peak) [13]. The tremor frequency range of a normal person does not differ from the one of people suffering from pathological conditions (only the amplitude does), which helps engineers develop some compact medical tools without taking another round of measurements. These devices are critical in special medical procedures such as brain tumour removal and ophthalmological microsurgery [14]. One example of a handheld active tremor compensation instrument called Micron [15] was developed by a group of researchers in Singapore.
This device was designed to improve the accuracy of surgeons in retinal surgery which include direct manipulation of vessels. The first iteration of Micron used piezoelectric actuator to position the tip of the instrument, independently of the body, controlled by a visual feedback loop (a LED attached to the tip and a PSD - Position Sensing Detector). Second iteration of Micron used an accelerometer to detect the tip movement and to estimate the hand tremor. Another mechatronic device [16] for microsurgeries uses bimorphs actuators and an IMU to position the instrument tip. There are some examples based on micromotors [17] connected into a Gough-Stewart platform configuration which provides six degrees of freedom and allow the device to position the tip efficiently and compensate the tremor on all DOF.

Previous research on literature did not present any approach over a laser surgical hand device that uses active cancelling tremor mechanisms. This subject will be discussed in this paper, presenting some active damping techniques for such devices.

\section{Theme Analysis}

This article analyses the operation of laser beam devices in microsurgery and analyses possible solutions to compensate for hand movement in order to stabilize the position of the laser beam on the surface of the object and to correct defocusing.

The solutions proposed in the scientific literature for tremor compensation in microsurgery consider the use of mechanical instruments (cutting, grasping, scissors). Mechanical methods require both the correct positioning of the instrument endpoint and sometimes the correction of orientation deviations (direction of approach to the target). The use of a laser scalpel only requires the position correction of the focus point (tilting the beam direction a few degrees leads to an elliptical focusing spot instead of the circular one but is not relevant to those dimensions).

Guidance solutions for mechanical microsurgery require the development of high forces operation and a structure with moderate elasticity. Conventional systems for mechanical microsurgery contain a compliant positioning system with a mobile platform on which the medical instrument clamp is attached.

This compliant system is operated with miniature actuators which leads to a contradiction between necessities: in order to avoid the use of too large actuators a reduced rigidity of the compliant system is recommended. At the same time, the compliant system also takes over the external mechanical loads that appeared as a result of the medical act (cutting, shearing, scraping, etc.) and must not move significantly under their action (theoretically this parasitic movement can be compensated by actuators but involves a system rapid response, from sensors, calculation to actuator response time). 
In laser microsurgery the situation is more convenient because the system has no mechanical loads in opposition during processing.

From the analysis of the operation of such systems several possible solutions were identified, based on optical or mechanical solutions.

Constructively, at the output of the optical fiber, the beam, having divergence, is collimated, then brought to a beam of parallel rays.

It is then passed through a focusing lens, which will be set in motion to counteract the translational movement of the hand.

\subsection{The Mechanical Solution}

It is based on the solidarity of the whole optical system (source, collection / collimation lens and, if it is necessary to increase the power, the focusing lens) in a single cylindrical assembly and its movement in space.

The practical solution is to create an actuation system with integrated elastic support system.

It can be done by:

1. Translation in the direction transverse to the beam axis; a single wide compliant system can be built to prevent the rotation of the mobile body, preferably with high rotational rigidity to ensure translation without the rotation of the laser source. An alternative is to use two compliant bearing planes with integrated actuators (Fig. 1). These planes could have a limited rotational elasticity, preferable with the same rotational virtual point for both systems, allowing control of transversal movements but also a limited control of rotations.

2. Translation in the transverse direction on the beam axis, only in one plane, and a compliant spherical joint (a rigid with a fixed point). The necessary rotation in the joint is a maximum of 3 degrees, which allows to achieve it by a compliant type solution. The compliant translation system must also ensure the possibility of small rotations, the virtual rotational point of this system being close to the rotational point of the spherical joint (Fig. 2).

3. We can also opt for a solution with a single compliant system if it is rigid enough to carry the laser source. A mobile platform solution with minimum two degrees of freedom (two rotations of the upper plate corresponding to the perpendicular axes of rotation in the plane of the platform) is used and eventually, for focusing, a translation in the direction of the axis of the whole system, perpendicular to the platform. The solution is similar to the systems used to reduce the effects of tremor in mechanical microsurgery [7] (Fig. 3).

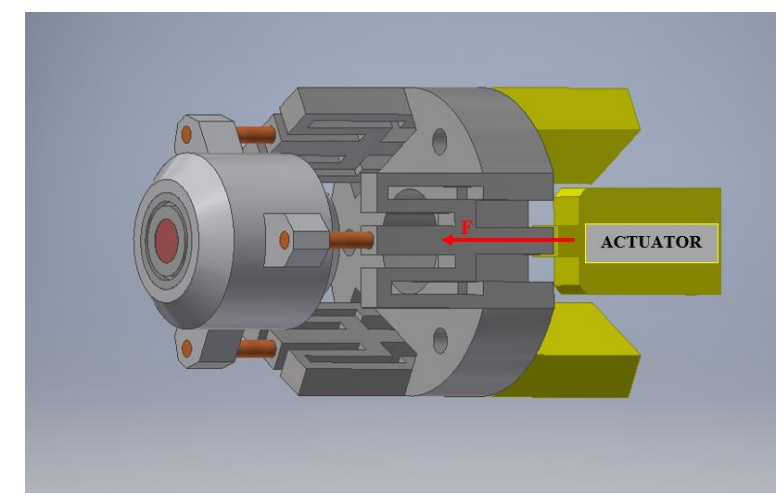

Figure 3: Platform example

The mobile system must allow the placement of the laser source, of about 50 grams, without deforming more than $5 \mu \mathrm{m}$ in transverse directions or rotating by more than 0.2 degrees, under the action of the weight of the laser source and its own weight, regardless of his position. Both in Figs. 1 as in Figs. 2, two actuators can be used (one in each direction, and an opposite elastic element) but due to the nonlinearity of the electromagnetic actuators, it is convenient in this case to use 4 actuators for each actuating plane (a pair for each direction).

Both figures 1 and 2 have placed the actuators in radial directions but the structure is modifiable, with the placement of the actuators in the axial direction and a mechanism for changing the direction of actuation. This last variant has the advantage of using longer length actuators, which leads to the higher displacements and forces (valuable for all common electrodynamic, electromagnetic and piezoelectric stack actuators).

The main problem with all solutions is related to the mutual interaction of the driving directions (moving in one direction also leads to the variation of the force in the other direction), which makes the control difficult.
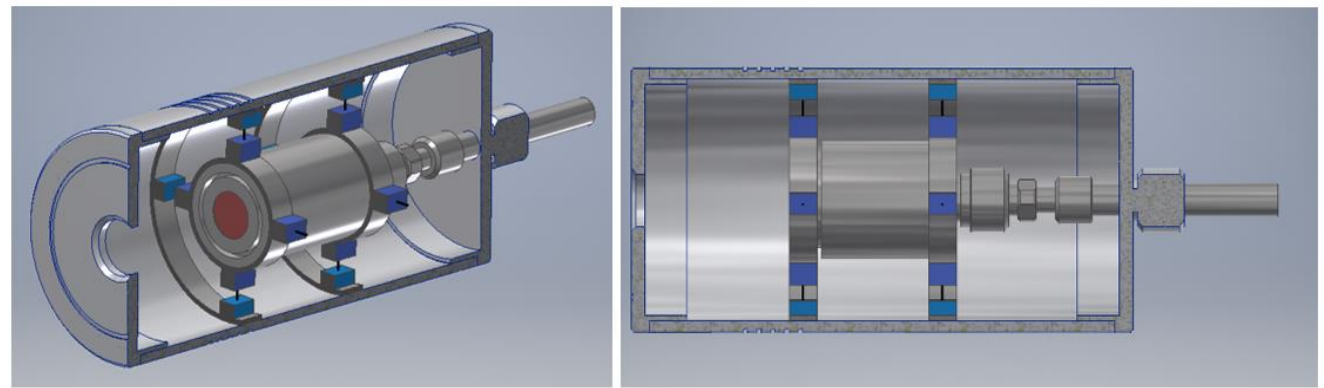

Figure 1: Drive solution with displacement of the entire laser source, with free rigid motion in space and tandem drive in two directions and in two planes 

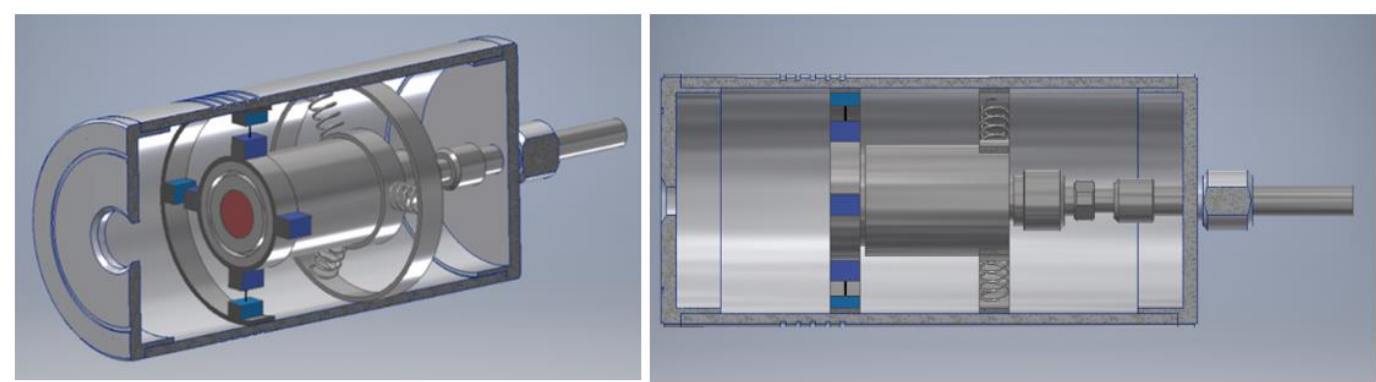

Figure 2: Drive solution with displacement of the entire laser source, with fixed point rigid motion, compliant rotational joint and tandem drive in two directions

\subsection{The Optical Solution}

These solutions consider the optical principles, as follows:

1. For a beam of rays parallel to each other and parallel to the optical axis, symmetrical to the optical axis or displaced to the optical axis, the focus is on the focal point image of the lens.

2. If a laser beam is parallel to the optical axis and the lens is translated perpendicular with the optical axis, the image focal point is also translated.

3. The translation of the lens in two directions in its plane leads to the translation of the focusing spot. The translation of the lens in the direction of its axis leads to the correction of defocus.

4. If the beam of parallel rays is inclined in relation to the optical axis, the focusing is made at a point in the image focus plane, defined as the plane perpendicular to the optical axis, and passing through displaced in respect to the point $\mathrm{F}^{\prime}$.

The inclination of the beam can be achieved using a controlled rotating mirror or a Risley prism. Placing a prism in the path of a beam parallel to the optical axis will obtain a rotation of the beam and a translation of the focus point in the image focus plane.

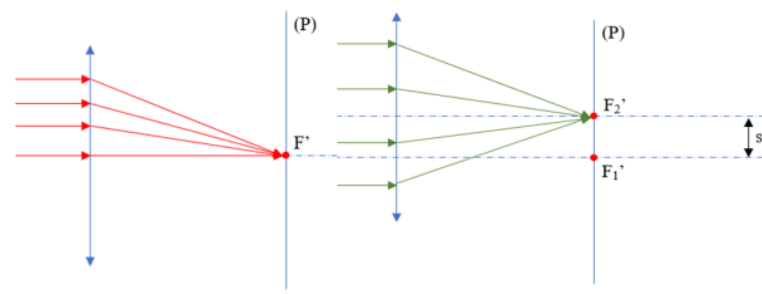

Figure 4: Lens image formation during translation

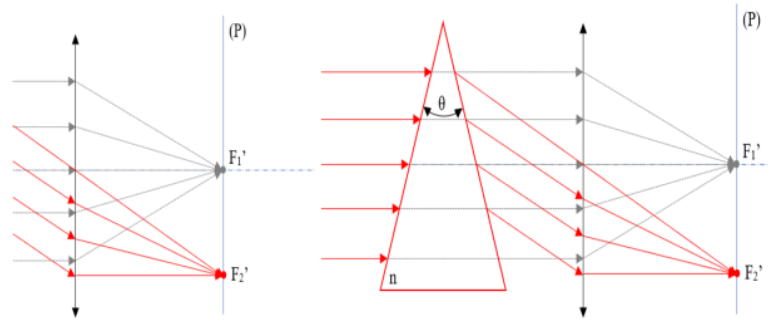

Figure 5: Image formation through the lens at beam rotation
The technique is more difficult to use in offset conditions because a variable angle prism is required. It is possible to make such a prism (it is made of two halves of the cylinder, cut obliquely relative to the axis of the cylinder, and their rotation in opposite directions, with equal angles). To obtain a two-way rotation, the rotation of the cylinder axis is also necessary (Figure 6). The solution is more complex but possible.

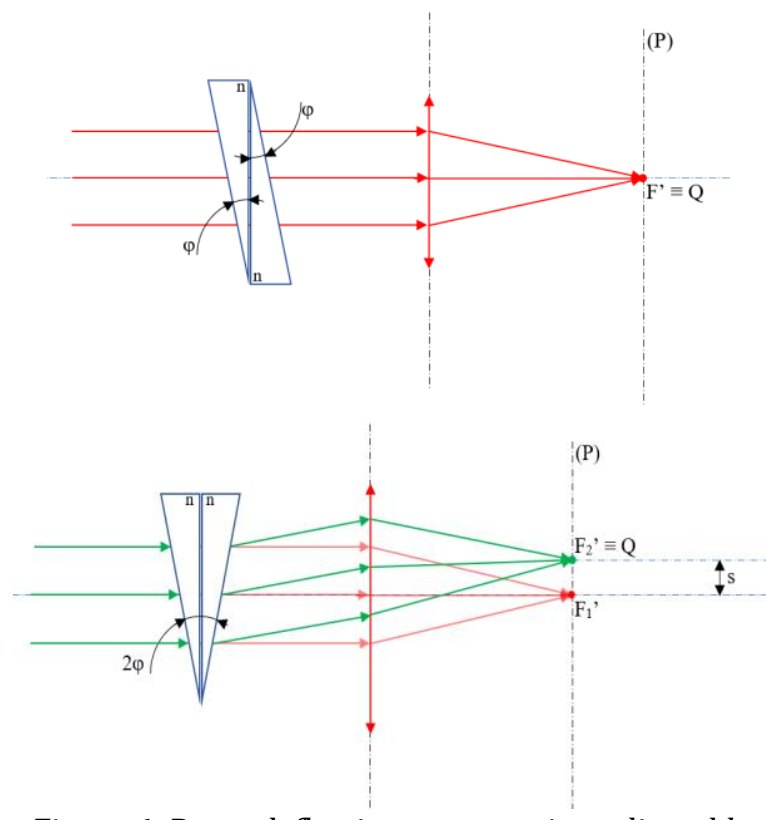

Figure 6: Beam deflection system using adjustable Risley prism

\section{- What happens if the lens is rotated?}

The rotation of the lens leads to the rotation of the image focus plane. The horizontal ray passing through the centre of the lens, which is initially on the optical axis, will pass undisturbed and focus on a point in the image focus plane. But the image focus plane is rotated with the lens. Consequently, a beam defocusing will occur but without moving the spot in the noted target plane notated with (P1).

The beam creates in the plane (P1) a spot in the same position as the initial focus point, but elliptical and not circular because the beam of rays in the image plane no longer forms a cone with circular symmetry with respect to the previous optical axis (it was assumed that the initial beam is cylindrical 
and has the optical axis as the axis of symmetry) (Figure 7).

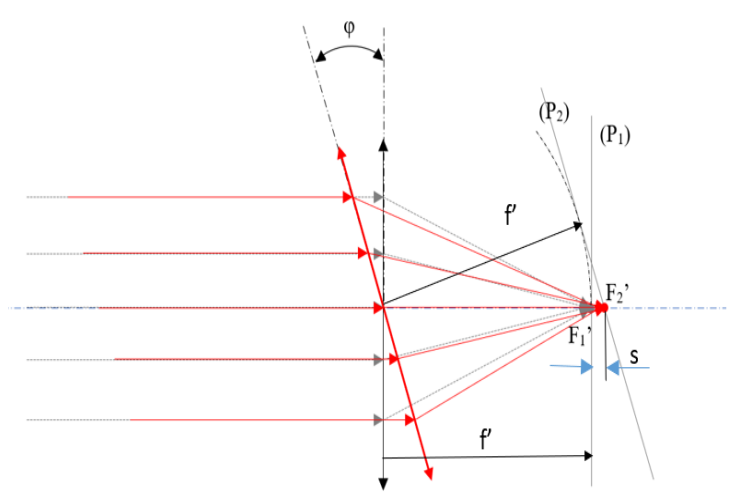

Figure 7: Modification of a beam of parallel rays as it passes through a rotating converging lens

The maximum diameter of the light spot that appears in the horizontal plane can be described by the following equation:

$$
\begin{gathered}
D=2 * s * \operatorname{tg}(\theta)=2 * \operatorname{tg}\left(\frac{h}{f^{\prime}}\right) * s \\
D=2 * s * \operatorname{tg}(\theta)=2 * s * \operatorname{tg}\left(\frac{h}{f^{\prime}}\right) \\
D=2 * s * \operatorname{tg}(\theta)=2 * \operatorname{tg}\left(\frac{h}{f^{\prime}}\right) * s
\end{gathered}
$$

where $\mathrm{s}$ is the defocus distance which can be calculated at big angles from the equation 2 .

$$
\begin{gathered}
f^{\prime}=\left(s+f^{\prime}\right)^{*} \cos (\varphi) \\
s=f^{\prime}\left(\frac{1}{\cos (\varphi)}-1\right)= \\
=f^{\prime}\left(\begin{array}{l}
1+\left.\frac{\sin (\varphi)}{\cos ^{2}(\varphi)}\right|_{\varphi=0} \varphi+ \\
\left.\frac{\cos ^{3}(\varphi)+2 \cos (\varphi) \sin ^{2}(\varphi)}{\cos ^{3}(\varphi)}\right|_{\varphi=0} \varphi^{2}+\ldots-1
\end{array}\right)
\end{gathered}
$$

For small angles, the equation becomes:

$$
S \cong f^{*} \varphi^{2}
$$

The diameter of the light spot becomes:

$$
D=2 * \operatorname{tg}\left(\frac{h}{f^{\prime}}\right) * f^{\prime} * \varphi^{2}
$$

The usual focal length is of the order of $100 \mathrm{~mm}$ (in order to allow the doctor to visualize the result) and the rotation of the hand is expected, according to current studies, to be less than 5 degrees, maximum 10 degrees (in medical cases), which leads to a spot defocusing of $s \cong 3.1 \mathrm{~mm}$ and $\mathrm{D}=3.24 \mathrm{~m}$.

If a $6 \mathrm{~mm}$ diameter beam is considered, results a sufficiently small spot diameter that will not have any significant impact.

Consequently, the rotation of the lens does not introduce any meaningful effects to the current application. Defocus due to rotation can be counteracted by an axial displacement of value $s$, which brings the focus point to the target plane.

- What happens if the lens translation and rotation are combined?

If the translation is made in the direction of the optical axis simultaneously with the rotation, a double defocusing will occur (due to the translation on the optical axis, with the effect of a circular spot instead of the initial spot, and then an elliptical shape due to the asymmetric cone of rays, occurred as a result of rotation).

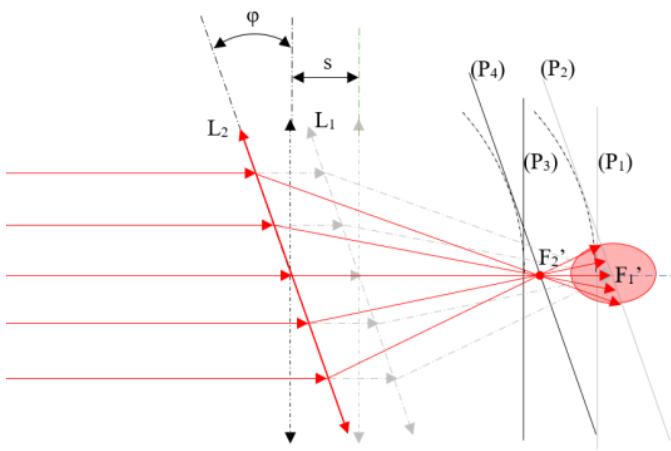

Figure 8: Focusing a beam as it passes through a rotating and axially translated converging lens

Figure 8 shows: (P1) the initial focal plane, (P2) the focal plane due only to rotation, (P3) the focal plane due only to axial translation, (P4) the focal plane due to overlap and rotation. It can be corrected by moving the lens in the direction of the optical axis, with a value equal to that of the axial defocus minus the micro-displacement of rotation correction.

- How to correct the movements of the whole device (including the laser source)?

The complex movement of the entire device (of the beam but also of the lens) leads to defocusing combined with the translation of the light spot. Rotating the lens with the same value as the hand but in the opposite direction can lead to the return of the image focus plane parallel to the work plane but does not solve the defocus and does not solve the problem of transverse movement of the light spot in the work plane.

The applicable solution is to move the lens in its holder (in a direction perpendicular to the optical axis), until the optical axis passes through the 
desired focus point, doubled by an axial displacement (in the direction of the optical axis), until full focus is obtained (Figure 9).

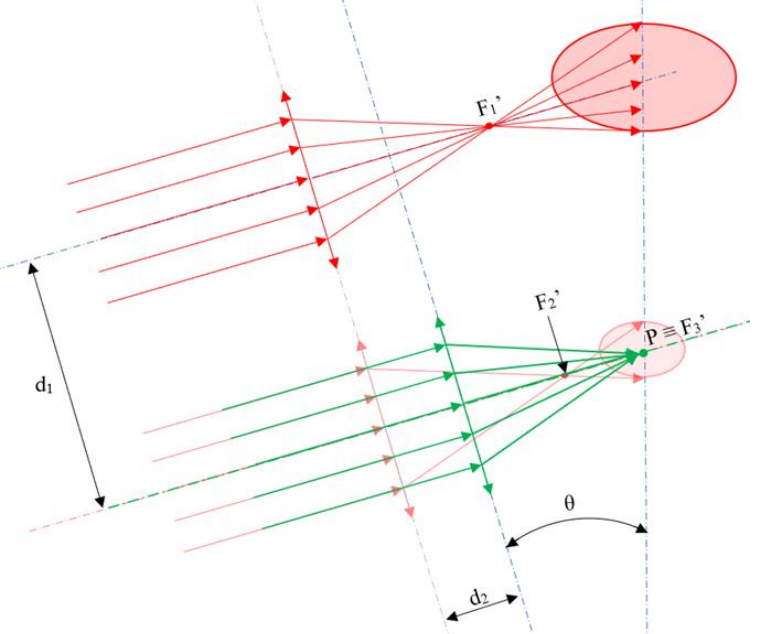

Figure 9: The effect of device rotation and translation is countered by a transversal and an axial displacement of the focusing lens

The required displacements, $d_{1}$ and $d_{2}$, are determined depending on the position of the optical system in space. For a uniform approach in the mechanical and optical case, a virtual terminal point of the microsurgery tool can be considered right in the image focal point of the lens. The analysis of the movements of the operator's hand determines its movements $\mathrm{a}_{1}$ and $\mathrm{a}_{2}$ but also the rotation $\theta$.

The necessary controls are determined by the relationships:

$$
\begin{aligned}
& d_{1}=a_{1} * \sin (\theta)+a_{2} * \cos (\theta) \\
& d_{2}=a_{1} * \cos (\theta)+a_{2} * \sin (\theta)
\end{aligned}
$$

Considering the aforementioned aspects, we can conclude the following:

- The translation of the operator's hand in directions ortogonal to the optical axis can be compensated by counter-movement in the same direction, identical as value but in the opposite sense of the focusing lens.

- The translation of the operator's hand in the direction of the optical axis can be compensated by the counter-movement in the same direction, identical as value but in the opposite sense of the focusing lens.

- When the rotation of the device occurs, a functional solution is that based on the translation of the lens in directions perpendicular to the axis of the device, until the optical axis passes through the desired focus point on target plane. It is necessary to perform small axial movements to restore focus and obtain a concentrated spot. The beam will pass through the edge of the lens, which can cause problems if there are significant optical aberrations.
If the rotations of the device are neglected and only the transverse displacements are considered, the transverse translation of the lens is sufficient for compensation. If the translation of the device in the direction of the optical axis is also considered, a lens focus translation is required.

If lens movement is not allowed, only a prism or mirror can partially solve the problem by directing the beam at a different angle at the lens so that the optical axis deviates in the image plane pinting to the target (Figure 10). The optical beam remains defocused and requires axial displacement of the lens.

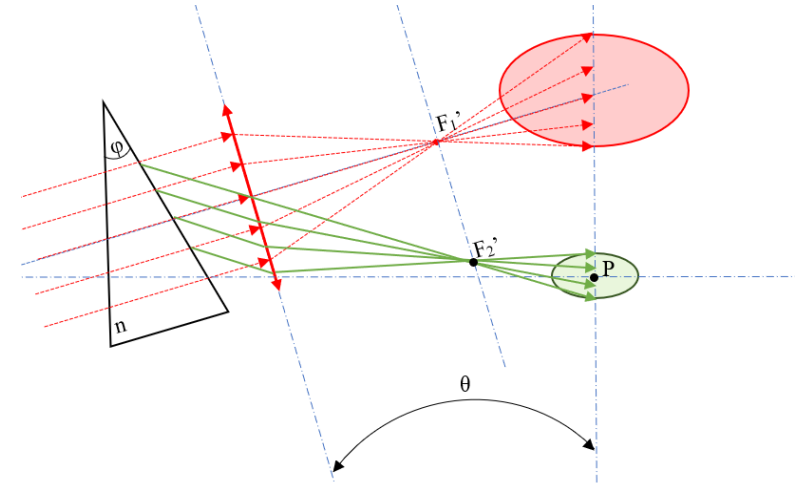

Figure 10: Use of a prism to partially correct the combined movement of the device

A complete compensation of the compound motion in the plane can be obtained if a prism combined with a displacement in the direction of the optical axis of the lens is used (Figure 11). The movement in the direction of the optical axis and the power of the prism (given by the peak angle of the prism) form a set of parameters that must be adjusted simultaneously. If the axial movement of the lens is easier, however, varying the power of the prism is difficult and involves a Risley prism or a modern solution based on adaptive optics.

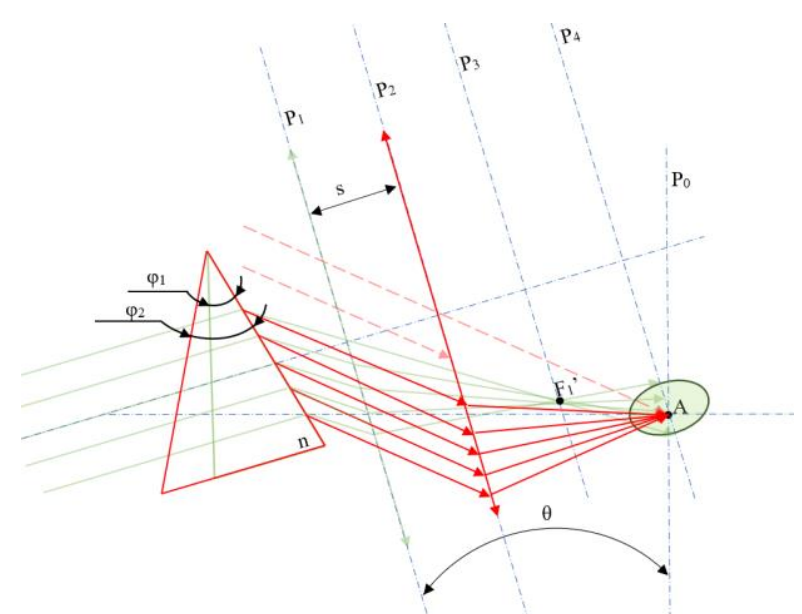

Figure 11: Use of a prism and optical axis translation for partial correction of the combined movement of the device 
An alternative to using a movable focusing lens is to place a double-rotating mirror in the path of the parallel beam. The beam will enter the focusing lens at an angle equal to twice the rotation angle of the mirror and will be focused in the image focal plane of the lens but with the focus point offset from the image focus point (Figure 12). The major problem with rotating the device assembly is that the image focal plane is rotated and translated also.

Two optical correction strategies are possible:

1. Focusing at the point determined by the intersection of the image focal plane with the target plane.

2. Focusing so that the main direction of the refracted beam is directed to the desired point in the target plane.

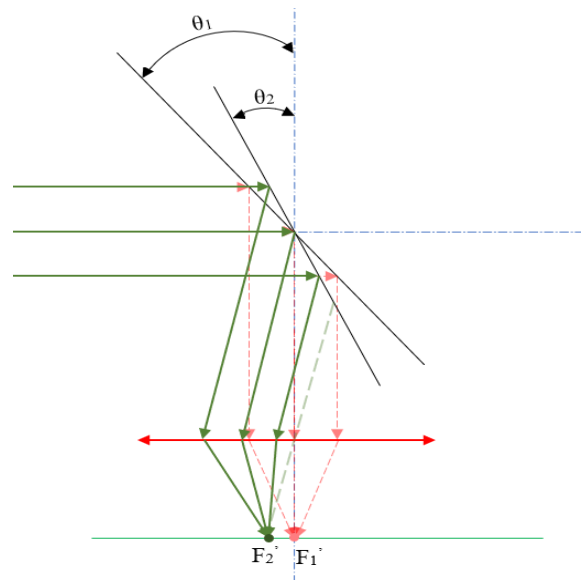

Figure 12: Use a mirror to correct the transverse movement of the device

In the first case, a well-focused image is obtained, but most likely at a different point from the desired one. In the second case, a defocused image is obtained but at the desired point in the image plane (correctable situation with a movement of the lens in the direction of the optical axis). In both cases only a deflection mirror or an adjustable prism is required. Unpleasant situations (correctly positioned but defocused spot or spot focused but displaced in the target plane) are solvable if an axial translation of the focusing lens occurs until the condition that the image focus plane intersects the target plane at the desired focus point, similar to using the prism. If only transverse translations of the device are considered, the resulting problem can be corrected solely by rotating the incident beam. Axial translation with defocus cannot be corrected by mirrors.

The rotation of the incident beam, with the help of adjustable mirrors or prisms, easily corrects the transverse translations of the device and can reduce the effect of the device rotations. Complete elimination of the effect of rotations as well as elimination of defocus are obtained only by adding a translation of the lens on its optical axis.

\section{Conclusions}

In conclusion, the development of a manual laser device for microsurgery operations can be approached from at least two perspectives: mechanical or optical. Due to the increased precision required by the application, for both cases it is necessary to define the optimal operating parameters, namely the amplitude and direction of hand tremor.

According to those presented, three constructive solutions for the mechanical approach were identified: the solution with the mobile platform (two rotations in the platform plane and a translation); the solution with a rigid spherical articulated body (two rotations relative to the articulation point) and a drive solution with displacements of the entire laser source, with free rigid motion in space.

It was observed that in the case of mechanical solutions, they have a larger size due to the integral displacement of the optical assembly (source, collection / collimation lens and the focusing lens) located on the compliant system. In this case, the static deformation (or rotation) of the mobile subassembly of the active damping system should not exceed a value of $5 \mu \mathrm{m}$ (or 0.2 degrees) regardless of its position. Due to the approximate weight of $50 \mathrm{~g}$ of the mobile subassembly, a dynamic analysis of the system is necessary in order to be able to choose the optimal actuators in terms of actuating force but also of the gauge.

These aspects represent the hypotheses for the next stages of research.

For the optical approach of the correction system, the solutions are multiple, using mobile focusing lenses, variable angle prisms (Risley prism) or movable mirrors. The paper presents the limitations and advantages of each solution for complete correction in any possible cases of hand tremor and orientation.

The translations of the laser device on the optical axis direction or perpendicular on this axis can be corrected by translating the lens, which will move the image focus point and match the focal image point with the target point.

To compensate for device rotations, multiple movements are required, such as: lens translations in three directions (xyz plane of the lens) or beam deflection at a certain angle and one axial translation. If the rotations of the device are neglected and only the transverse displacements are considered, the transverse translation of the lens is sufficient for compensation.

Designing an adjustable optical system and a compliant device that it is able to position a lens to counter the hand tremor represent the next research step for the development of a manual laser device with active tremor compensation. 


\section{Acknowledgements}

This work was supported by a grant of the Romanian Ministry of Education and Research, CCCDIUEFISCDI, project number PN-III-P2-2.1-PTE-20190253, within PNCDI III.

\section{References}

[1] A. Anouti și W. C. Koller, „Tremor disorders. Diagnosis and management.", West. J. Med., vol. 162, nr. 6, pp. 510-513, iun. 1995.

[2] K. P. Bhatia et al., „Consensus Statement on the Classification of Tremors. From the Task Force on Tremor of the International Parkinson and Movement Disorder Society", Mov. Disord. Off. J. Mov. Disord. Soc., vol. 33, nr. 1, pp. 75-87, ian. 2018, doi: $10.1002 /$ mds.27121.

[3] P. Bain, „A combined clinical and neurophysiological approach to the study of patients with tremor.", J. Neurol. Neurosurg. Psychiatry, vol. 56, nr. 8, pp. 839-844, aug. 1993.

[4] G. Deuschl, M. Lauk, și J. Timmer, „Tremor classification and tremor time series analysis”, Chaos Interdiscip. J. Nonlinear Sci., vol. 5, nr. 1, pp. 48-51, mar. 1995, doi: 10.1063/1.166084.

[5] A. T. Winfree, The Geometry of Biological Time. Springer Science \& Business Media, 2001.

[6] Wei Tech Ang, M. Krichane, și Terence Sim, „Zero Phase Filtering for Active Compensation of Periodic Physiological Motion", în The First IEEE/RAS-EMBS International Conference on Biomedical Robotics and Biomechatronics, 2006. BioRob 2006., Pisa, Italy, 2006, pp. $182-$ 187, doi: 10.1109/BIOROB.2006.1639081.

[7] C. N. Riviere, Wei Tech Ang, și P. K. Khosla, „Toward active tremor canceling in handheld microsurgical instruments", IEEE Trans. Robot. Autom., vol. 19, nr. 5, pp. 793-800, oct. 2003, doi: 10.1109/TRA.2003.817506.

[8] M. U. Humayun, R. S. Rader, D. J. Pieramici, C. C. Awh, și E. de Juan, „Quantitative Measurement of the Effects of Caffeine and Propranolol on Surgeon Hand Tremor", Arch. Ophthalmol., vol. 115, nr. 3, pp. 371-374, mar. 1997, doi: 10.1001/archopht.1997.01100150373010.

[9] Denis Huen, Jindong Liu, and Benny Lo, “An Integrated Wearable Robot for Tremor Suppression with Context Aware Sensing", IEEE 13th International Conference on Wearable and Implantable Body Sensor Networks (BSN), 2016, Electronic ISSN: 2376-8894
[10] E. Rocon, J. M. Belda-Lois, A. F. Ruiz, M. Manto, J. C. Moreno, and J. L. Pons, "Design and Validation of a Rehabilitation Robotic Exoskeleton for Tremor Assessment and Suppression", IEEE Transactions on Neural Systems and Rehabilitation Engineering, Vol. 15, No. 3, September 2007, DOI: 10.1109/TNSRE.2007.903917

[11] D. Huen, J. Liu, and B. Lo, "An Integrated Wearable Robot for Tremor Suppression with Context Aware Sensing", IEEE 13th International Conference on Wearable and Implantable Body Sensor Networks (BSN), San Francisco, CA, USA, 14-17 Iunie 2016, DOI: 10.1109/BSN.2016.7516280

[12] Ryden LE, Matar E, Szeto JY, Hammond DA, Clouston P, Lewis SJ. "Shaken not stirred: A pilot study testing a gyroscopic spoon stabilization device in Parkinson's disease and tremor", Ann Indian Acad Neurol 2020; doi:10.4103/aian.AIAN_251_19

[13] Yue Zhou, Mary E. Jenkins, Michael D. Naish, and Ana Luisa Trejos, "Development of a Wearable Tremor Suppression Glove", 2018 7th IEEE International Conference on Biomedical, Enschede, The Netherlands, August 26-29, 2018. DOI: 10.1109/BIOROB.2018.8487197

[14] Wei Tech Ang, M. Krichane, și Terence Sim, „Zero Phase Filtering for Active Compensation of Periodic Physiological Motion", în The First IEEE/RAS-EMBS International Conference on Biomedical Robotics and Biomechatronics, 2006. BioRob 2006., Pisa, Italy, 2006, pp. 182187, doi: 10.1109/BIOROB.2006.1639081

[15] Robert MacLachlan, Brian Becker, Jaime Tabares, Gregg Podnar, Louis Lobes and Cameron Riviere, "Micron: An Actively Stabilized Handheld Tool for Microsurgery", IEEE TRANSACTIONS ON ROBOTICS, VOL. 28, NO. 1, February 2012, DOI: 10.1109/TRO.2011.2169634

[16] W.T. Latt, U-X. Tan, C. Y. Shee, C. Riviere and W. T. Ang, "Compact Sensing Design of a Handheld Active Tremor Compensation Instrument", IEEE SENSORS JOURNAL, VOL. 9, NO. 12, December 2009, DOI:10.1109/JSEN.2009.2030980

[17] H. Kamble, K. Masurkar, B.B. Ahuja, E. Kulkarni, "Mechatronics Device for Tremor Sensing and Cancellation for Accuracy Enhancement in Microsurgeries", IEEE International Conference on Advances in Engineering \& Technology Research (ICAETR-2014), August 01-02, 2014, India, DOI:10.1109/ICAETR.2014.7012806 